\title{
Clinical Outcome of Arthroscopic Debridement for Septic Arthritis of Shoulder
}

\begin{abstract}
Keywords: Arthroscopic debridement; Septic arthritis of the shoulder Abstract

Background: Septic arthritis of the shoulder is relatively rare; therefore, few reports have addressed the associated clinical outcomes. The purpose of the present study was to investigate the c linic al outc omes of arthrosc opic debridement in patients with septic arthritis of the shoulder.

Methods: Fifteen patients with septic arthritis of the shoulder were treated at our institution between 2003 and 2011. Of these, we examined 10 patients ( 8 men and 2 women) who underwent arthroscopic debridement with the average follow-up period of 52.2 months (12-120 months). The average age at the onset was 65.6 years, ranging from 36 to 90 years. The average time from the onset to the surgery was 12 days (4-25 days). The Gächter classification was used for intraoperative assessment and the Japanese Orthopaedic Association (J OA) score was for postoperative assessment.
\end{abstract}

Results: All the patients had the underlying complications before surgery: hypertension, diabetes mellitus, liver dysunction or meumatoid arthritis. Intra-articular steroid injection was performed in 5 patients. The causative organism was Staphylococcus aureus in 4 patients, methicillin-resistant S. aureusin 4 patients, and unknown in the remaining 2 patients. Intra oparative evaluation using the Gächter classification system demonstrated 3 patients with stage I, 2 with stage II, 3 with stage III, and 2 with stage IV. In the 2 patients with stage IV humeral head resection was perfomed within 1 year after the index surgery.

Conclusions: The 2 patients with the Gächterstage IV who underwent bone resection after arthroscopic debridement had prolonged time from the onset to the surgery, compared with the other succ essful cases. These results therefore indicate the importance of arthroscopic surgery performed in early stage of the disease.

\section{Background}

Septic arthritis of the shoulder is a relatively rare, and accounts for approximately $10 \%$ in the septic arthritis [1,2]. The elderly and immunocompromised patients are reported to be susceptible to this disease [3,4]. The treatments include antibiotic administration, puncture, incision/drainage, and open/arthroscopic surgery [4]. Arthroscopic debridement, a less invasive technique, has been performed in patients with the septic arthritis of the shoulder [1,4] however, few reports have addressed the clinical outcome in this disease. The purpose of the present study was to investigate the clinical outcomes of arthroscopic debridement in patients with septic arthritis of the shoulder.

\section{Methods}

We examined 10 shoulders (4 right and 6 left shoulders) of 10 patients ( 8 men and 2 women) with septic arthritis who underwent arthroscopic debridement in our institution between 2003 and 2010. The average age at the onset was 65.6 years (36-90 years). All of our case was referred to the other clinic/hospital, or had severe

\section{Journal of}

Orthopedics \& Rheumatology

\section{Tsunemasa Matsubara ${ }^{1}$, Masafumi Gotoh ${ }^{1 *}$, Yasuhiro Mitsui ${ }^{1}$, Isao Shirachi ${ }^{1}$, Takahiro Okawa ${ }^{1}$, Fujio Higuchi ${ }^{1}$, Masahiro Shirahama ${ }^{1}$ and Naoto Shiba $^{2}$}

${ }^{1}$ Department of Orthopedic Surgery, Kurume University Medical Center, Fukuoka, Japan

${ }^{2}$ Department of Orthopedic Surgery, Kurume University, Fukuoka, Japan

\section{*Address for Correspondence}

Masafumi Gotoh, MD, PhD, Department of Orthopedic Surgery, Kurume University Medical Center, 155 Kokubu-machi, Kurume, Fukuoka 8390863, Japan, Tel: +81-942-22-6111 ex. 533; Fax: +81-942-22-6657; E-mail: gomasa@med.kurume-u.ac.jp

Submission: 21 November 2014

Accepted: 15 December 2014

Published: 20 December 2014

Copyright: @ 2014 Matsubara T, et al. This is an open access article distributed under the Creative Commons Attribution License, which permits unrestricted use, distribution, and reproduction in any medium, provided the original work is properly cited.

Reviewed \& Approved by: Dr. Elliott H. Leitman, Director of Orthopaedic Education, Christiana Care Health System, USA

complication that needed general management preoperatively; therefore, the average time to the surgery was 12 days (4-25 days). The average follow-up period was 52.2 months (12-120 months). In our series, 7 of 10 patients had shoulder injection before onset; however, we had no exact information regarding the time between injection and onset of infection. Institutional Review Board approval was obtained for this study (\#14052).

During arthroscopy, the patients were placed in the beach chair position. Conventional 3 portals (anterior, posterior, and lateral) were made on the shoulder involved. Thorough glenohumeral and subacromial debridement including synovectomy was performed during operation. After the surgery, closed-suction drain was placed at both the glenohumeral joint and the subacromial bursa until the amount of the drainage was less than $50 \mathrm{ml} /$ day (7-14 days). At least 5 litter of fluid was used during arthroscopic sIntra-venous antibiotic administration was used immediately after the surgery for 2 weeks, depending on the sensitivity of the pathogenic bacterium. Subsequent oral medication was continued until C-reactive protein level was normalized.

Intraoperative evaluation was performed using the Gächter classification system [5]: stage I indicates opacity of fluid, regardless of the synovial membrane, and possible petechial bleeding; stage II includes purulent material, severe inflammation and fibrinous deposition; stage III has thickening of the synovial membrane with cartilage erosion; stage IV is the most aggressive of the stages, with subchondral delamination. The Japanese Orthopaedic Association (JOA) score was used for postoperative assessment.

\section{Results}

All the patients had the underlying complications before surgery: hypertension, diabetes mellitus and liver dysfunction and rheumatoid 
Citation: Matsubara T, Gotoh M, Mitsui Y, Shirachi I, Okawa T, et al. Clinical Outcome of Arthroscopic Debridement for Septic Arthritis of Shoulder. J Orthopedics Rheumatol. 2014;2(1): 3.

ISSN: $2334-2846$

Table 1: Case information.

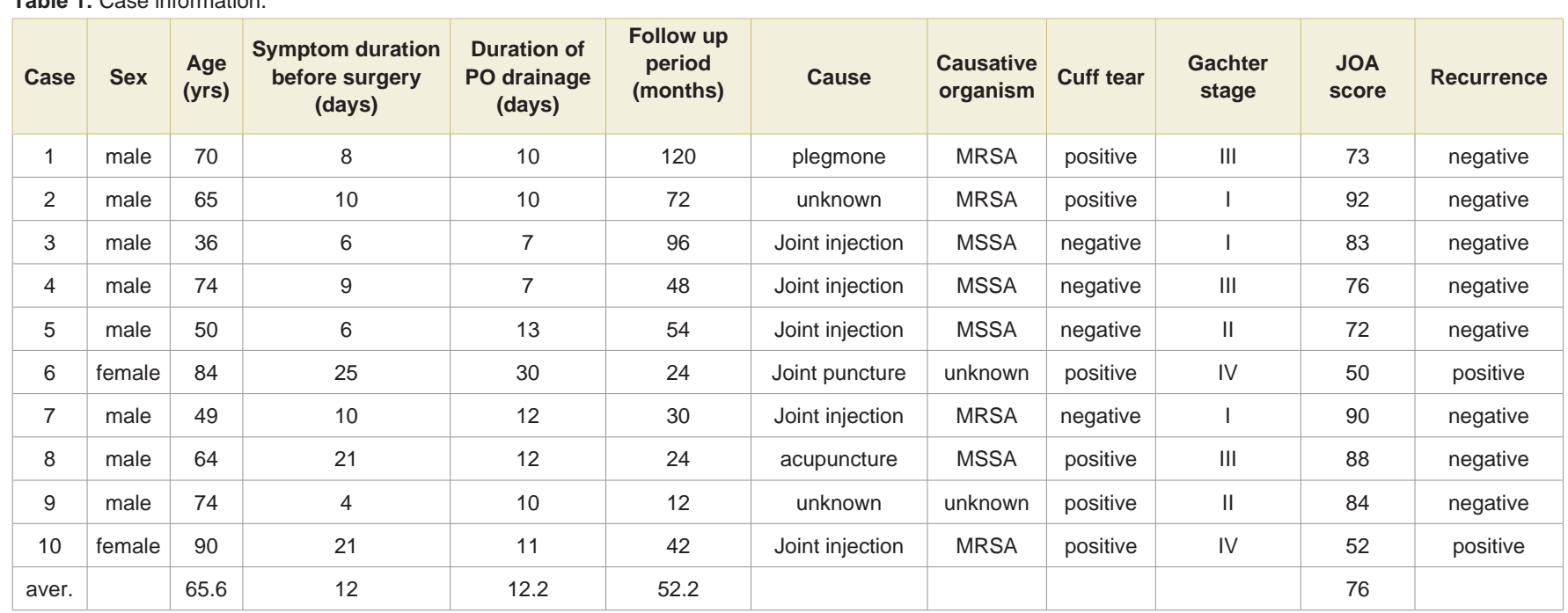

*MRSA: Methicillin-resistant Staphylococcus aureus; MSSA: Methicillin-susceptible Staphylococcus aureus

arthritis. Intra-articular steroid injection was performedin 5 patients. The causative organism was methicillin-susceptible Staphylococcus aureus (MSSA) in 4 patients, methicillin-resistant S. aureus (MRSA) in 4 patients, and unknown in the remaining 2 patients.

\section{Intraoperative assessment}

According to the Gächter classification, 3 patients were classified as stage I, 2 as stage II, 3 as stage III, and 2 as stage IV. Rotator cuff tear was noted in 6 patients.

\section{Postoperative assessment}

Postoperative radiographs indicated bone resorption of the greater tuberosity with degenerative changes of the glenohumeral joint in 7 patients, of whom 4 had no abnormalities on preoperative radiographs.

The mean time for normalization of C-reactive protein level was 47.4 days (14-120 days). The average postoperative JOA score was 76 points (73-92 points). Recurrence of the septic arthritis was observed in 2 patients with the Gächterstage IV, each of whom underwent bone resection at 90 days and 74 days from the index surgery.

These data are summarized in Table 1.

\section{Discussion}

Although septic arthritis of the shoulder is rare, secondary arthritic changes (cartilage destruction and osteonecrosis) are induced in cases with the delayed diagnosis [1], causing fatal septic shock due to bacteremia [6]: Elderly individuals, as well as immunocompromised patients, are susceptible to this disease [7]. Similarly, the preoperative underlying complications were noted in all 10 patients in the present study.

Intra-articular injection is considered to be the most associated causes of the septic arthritis of the shoulder $[1,3,8]$. In the present study, 6 of 10 patients hadintra-articular injection; of these, steroids were used in 5 patients.

Stutz et al. reported that in septic arthritis of the knee, re- surgery was needed in $>50 \%$ of the patients with the Gächterstage II or higher [5]. In the present study, 2 patients with stage IV needed revision surgery, but not in 5 patients with stage II or III. In-Ho et al. reported that arthroscopic surgery performed within 2 weeks from onset yielded favorable outcomes in septic arthritis [1]. Actually in the 2 patients with stage IV, the time from the onset to the surgery was more than 2 weeks ( 25 and 21 days, respectively), in contrast to within 2 weeks in patients with stage II or III. Therefore, we believe that early surgical intervention contributes to the favorable outcome in patients with septic arthritis of the shoulder.

Noteworthy, one patient with the Gächter stage III did not progress recurrent, despite having longer time before the surgery (21 days). This patient with diabetes mellitus, who had poor glycemic control, had undergone an incision/drainage at 11 days from the onset, before referred to our institution. Once the blood glucose level was well controlled, arthroscopic surgery was performed, leading to a favorable outcome. Thus, incision/drainage within 2 weeks from onset may have been effective not to aggravate septic arthritis in this case.

Two patients with the Gächter stage IV who had the humeral head destruction on preoperative radiographs underwent humeral head resection after arthroscopic debridement. In such cases, invasive open surgery would be first-choice instead of arthroscopic treatment.

\section{Conclusion}

In the present study, we examined 10 patients with septic arthritis of the shoulder who underwent arthroscopic debridement. Recurrence was observed in 2 patients who had time of $>2$ weeks before surgery and the Gächter stage IV during arthroscopy. Thus, we strongly suggest that arthroscopic debridement in early stage plays an important role in obtaining successful outcome in septic arthritis of the shoulder.

\section{References}

1. Jeon IH, Choi CH, Seo JS, Seo KJ, Ko SH, et al. (2006) Arthroscopic management of septic arthritis of the shoulder joint. J Bone Joint Surg Am 88 1802-1806. 
Citation: Matsubara T, Gotoh M, Mitsui Y, Shirachi I, Okawa T, et al. Clinical Outcome of Arthroscopic Debridement for Septic Arthritis of Shoulder. J Orthopedics Rheumatol. 2014;2(1): 3.

2. Ito $O$ (2011) The clinical results for pyogenic arthritis of the shoulder: report of 5 cases. Katakansetsu 35: 931-934.

3. Oshima M, Nakagawa Y, Tomita K (2004) Treatment of pyogenic arthritis of the shoulder. Katakansetsu 28: 315-320.

4. Abdel MP, Perry KI, Morrey ME, Steinmann SP, Sperling JW, et al (2013) Arthroscopic management of native shoulder septic arthritis. J Shoulder Elbow Surg 22: 418-421.

5. Stutz G, Kuster MS, Kleinstück F, Gächter A (2000) Arthroscopic management of septic arthritis: stages of infection and results. Knee Surg Sports Traumato Arthrosc 8: 270-274.
6. Esenwein SA, Ambacher T, Kollig E, Kutscha-Lissberg F, et al. (2002) Septic arthritis of the shoulder following intra-articular injection therapy. Lethal course due to delayed initiation of therapy [in German]. Unfallchirurg 105: 932-938.

7. Cleeman E, Auerbach JD, Klingenstein GG, Flatow EL (2005) Septic arthritis of the glenohumeral joint: a review of 23 cases. J Surg Orthop Adv 14: $102-$ 107.

8. Shemesh S, Heller S, Salai M, Velkes S (2011) Septic arthritis of the knee following intraarticular injections in elderly patients: report of six patients. Isr Med Assoc J 13: 757-760.

\section{Acknowledgements}

We thank Hidehiro Nakamura, MD from the Department of Orthopaedic Surgery, Kurume University for his help with the preparation of this manuscript. 